\title{
Negative Regulation of TSC1-TSC2 by Mammalian D-Type Cyclins
}

\author{
Sima J. Zacharek, ${ }^{1,2}$ Yue Xiong, ${ }^{1,2,3,4}$ and Stuart D. Shumway ${ }^{1}$ \\ ${ }^{1}$ Lineberger Comprehensive Cancer Center, ${ }^{2}$ Curriculum in Genetics and Molecular Biology, ${ }^{3}$ Department of Biochemistry and Biophysics, \\ and ${ }^{4}$ Program in Molecular Biology and Biotechnology, University of North Carolina, Chapel Hill, North Carolina
}

\begin{abstract}
The metazoan cell cycle is driven by the timely and composite activities of cyclin-dependent kinases (CDKs). Among these, cyclin D- and cyclin E-dependent kinases phosphorylate the pRb family proteins during $G_{1}$ phase of the cell cycle and thereby advance cells beyond the restriction point. Increasing evidence suggests that cyclin D-dependent kinases might affect events other than Rb pathway-mediated entry into $S$ phase, such as accumulation of cell mass. However, little is known about cyclin $D$ activity toward Rb-independent pathway(s) or non-pRb substrates. In this article, we show that the tumor suppressor TSC2 is a cyclin D binding protein. Coexpression of cyclin D1-CDK4/6 in cultured cells leads to increased phosphorylation and decreased detection of both TSC2 and TSC1, and promotes the phosphorylation of the mTOR substrates, 4E-BPI and S6K1, two key effectors of cell growth that are negatively regulated by the TSC1-TSC2 complex. At the cellular level, ectopic expression of cyclin D1 restores the cell size decrease caused by TSC1-TSC2 expression. Intriguingly, down-regulation of TSC proteins was also observed by the expression of a mutant cyclin D1 that is unable to bind to CDK4/6, or by the coexpression of cyclin D1 with either an INK4 inhibitor or with catalytically inactive CDK6, indicating that cyclin D may regulate TSC1TSC2 independently of CDK4/6. Together, these observations suggest that mammalian D-type cyclins participate in cell growth control through negative regulation of TSC1-TSC2 function. (Cancer Res 2005; 65(24): 11354-60)
\end{abstract}

\section{Introduction}

Studies in Saccharomyces cerevisiae that laid the groundwork for our understanding of the cell division cycle also established its critical link to cell growth-the accumulation of cellular mass $(1,2)$. Cell cycle control and cell growth control must be coordinately regulated to maintain homeostatic cell size, yet the two processes are separable (3). The use of temperature-sensitive cell division cycle mutants showed that yeast cells blocked from progressing through the cell cycle could still continue to increase in size. However, when cell growth was blocked by nutrient deprivation or by inactivating key biosynthetic genes, the cell cycle could no longer proceed (2). Although disruptions in the control of the cell cycle, and more recently of cell growth, have been widely recognized as major contributors to tumorigenesis $(4,5)$, the molecular mechanisms linking the two processes are not well understood.

Requests for reprints: Yue Xiong, CB7295, Lineberger Comprehensive Cancer Center, The University of North Carolina, Chapel Hill, NC 27599-7295. Phone: 919-9622142; Fax: 919-966-8799; E-mail: yxiong@email.unc.edu.

(C)2005 American Association for Cancer Research.

doi:10.1158/0008-5472.CAN-05-2236
Entry into the proliferative cell cycle and progression through the $\mathrm{G}_{1}$ phase is initiated by extracellular mitogenic signaling, which in mammalian cells leads to the synthesis of D-type cyclins. Two catalytic subunits, cyclin-dependent kinases 4 and 6 (CDK4/CDK6), can interact with any of three D-type cyclins (D1, D2, and D3) to form up to six distinct kinase holoenzymes (collectively referred to as cyclin Ds-CDK4/6). Although individual D-type cyclins, and to a lesser extent, CDK4 and CDK6, are expressed differentially in a tissue-specific manner, different cyclin Ds-CDK4/6 complexes are biochemically similar if not indistinguishable. CDK4/6 are negatively regulated by two families of CDK inhibitors, the INK4 (inhibitors of CDK4) family, which can bind CDK4/6 and prevent their association with D-type cyclins, and the CIP/KIP family, which can bind to and inhibit cyclin Ds-CDK4/6 and cyclin Es-CDK2 in a ternary complex (6). Active cyclin Ds-CDK4/6, followed by cyclin Es-CDK2, phosphorylate pRb and the two other pocket proteins, p107 and p130, thereby lifting repression of the E2F transcription factors, allowing passage through the restriction point in $G_{1}$, and permitting expression of genes necessary for DNA replication during $S$ phase.

Some aspects of cyclin D and CDK4/6 biology cannot be reconciled by their kinase activity toward $\mathrm{pRb}$ family proteins alone. For example, as some cells undergo senescence and permanently withdraw from the cell cycle, such as during myotube differentiation, the level of cyclin D in the cell actually accumulates rather than decreases (7). Furthermore, a cyclin D1 mutant ineffective at targeting $\mathrm{pRb}$ retains transforming ability in cooperation with Ras (8). In a similar vein, disruption of cyclin D-CDK4/6 function by $\mathrm{p}^{\text {INK4a }}$ overexpression in melanocytes exhibits phenotypically distinct consequences from pRb inactivation (9). Cyclin D1 and CDK4 are dispensable for proliferation in flies and mice, but do seem to play a role in growth control, as disruption of either cyclin D or CDK4 results in reduced cell and overall organism size (10-14). In fact, overexpression of cyclin D or CDK4 in flies leads to an increase in cell and organ size in a pRbindependent manner $(11,15)$. Genetic studies have suggested a role for cyclin D-CDK in cell growth control by acting upstream of Hif-1 prolyl hydroxylase and the mitochondrial ribosomal protein mRpL12 (16, 17).

Despite the broad effects of cyclin D-CDK on cell cycle and cell growth control, little is known about its substrates other than the pRb family. Recently, Smad3 was identified as a direct cyclin Ds-CDK4/6 substrate, linking cyclin D-CDK activity with another effector of proliferative control (18). In addition, CDK-independent activation of several transcription factors by cyclin $\mathrm{D}$ have been described (19). The identification of other cyclin D-CDK4/6 targets could lead to a greater understanding of its role in cell cycle progression, cell growth control, and tumorigenesis. In this study, we identify the cell growth regulator TSC2 as a cyclin D-binding protein. Further characterization of this interaction suggests that the TSC1-TSC2 complex may be a novel target of cyclin D-CDK4/6 activity involved in cell growth control. 


\section{Materials and Methods}

Mammalian cell culture, transfections, plasmids, and reagents. U2OS or HEK293T cells were grown at $37^{\circ} \mathrm{C}$ with $5 \% \mathrm{CO}_{2}$ in DMEM (Life Technologies, Gaithersburg, MD) supplemented with $10 \%$ fetal bovine serum (Sigma, St. Louis, MO), penicillin, and streptomycin (Invitrogen, San Diego, CA). Cells were transiently transfected using the calcium phosphate precipitation method or FuGene (Roche, Alameda, CA) according to the manufacturers' instructions. After transfection, cells were typically cultured for 24 to 48 hours prior to treatment or harvest for flow cytometry, immunoprecipitation, or Western analysis. To serumstarve cells, transiently transfected cells were cultured in DMEM overnight without serum (18 hours), followed by re-stimulation with $10 \%$ serum over a 4-hour time course. The cDNA encoding rat TSC2 was kindly provided by Dr. Ray Yeung (Department of Medical Genetics, University of Washington, Seattle, WA), and was subcloned into pcDNA3 in-frame with an HA-epitope tag. Flag-tagged human TSC2 was kindly provided by Dr. Lewis Cantley (Department of Systems Biology, Harvard Medical School, Boston, MA). Full-length cDNA encoding human TSCl was PCRamplified from a HeLa cDNA library and subcloned in-frame with a Myc-epitope tag. Constructs encoding HA-p70S6K and HA-4E-BP1 were kindly provided by Dr. Kun-Liang Guan (Department of Biological Chemistry, University of Michigan, Ann Arbor, MI) and Dr. John Blenis (Department of Cell Biology, Harvard Medical School, Boston, MA), respectively. Those encoding cyclins D1, D2, D3, E1, and A2, CDKs 1, 2, $2^{\mathrm{K} 33 \mathrm{M}}, 4,6,6^{\mathrm{K} 43 \mathrm{M}}$, and green fluorescent protein (GFP)-spectrin are from laboratory stock. Site-directed mutagenesis of cyclin D1 was done by standard PCR techniques using the QuikChange kit (Stratagene, La Jolla, CA). All constructs were verified by partial DNA sequencing.

Flow cytometry. Cells were transiently transfected with cDNAs of interest along with GFP-spectrin, cultured for 48 hours, collected by trypsinizing, washed and resuspended in cold PBS, and fixed by adding ethanol to a final concentration of $75 \%$. Fixed cells were resuspended in PBS/0.1\% Triton/0.1 mg/mL RNase, and DNA was labeled with propidium iodide $(1 \mathrm{mg} / \mathrm{mL})$ overnight at $4{ }^{\circ} \mathrm{C}$. Prepared cells were analyzed using a flow cytometer (FACScan, BD Biosciences, San Jose, CA), gating specifically on GFP-positive, transfected cells, using Summit software (version 3.0, BD Biosciences) for data processing.

Immunochemistry procedures and antibodies. Cells analyzed by Western blot or immunoprecipitation were lysed in NP40 lysis buffer [50 mmol/L Tris- $\mathrm{HCl}$ (pH 7.5), $0.15 \mathrm{~mol} / \mathrm{L} \mathrm{NaCl}, 0.5 \%$ Nonidet P- $40,1 \mathrm{mmol} / \mathrm{L}$ phenylmethylsulfonyl fluoride, $1 \mathrm{mmol} / \mathrm{L}$ DTT, $50 \mathrm{mmol} / \mathrm{L}$ sodium fluoride, $1 \mathrm{mmol} / \mathrm{L}$ sodium vanadate, and protease inhibitors: $2 \mu \mathrm{g} / \mathrm{mL}$ aprotinin, $2 \mu \mathrm{g} / \mathrm{mL}$ leupeptin, $10 \mu \mathrm{g} / \mathrm{mL}$ trypsin inhibitor, and $150 \mu \mathrm{g} / \mathrm{mL}$ benzamidine] and cleared by centrifugation. NP40-insoluble fractions were solubilized using an SDS lysis buffer [50 mmol/L Tris- $\mathrm{HCl}(\mathrm{pH} 7.5), 0.5 \mathrm{mmol} / \mathrm{L}$ EDTA, $1 \%$ SDS, $1 \mathrm{mmol} / \mathrm{L}$ phenylmethylsulfonyl fluoride, $1 \mathrm{mmol} / \mathrm{L}$ DTT, $50 \mathrm{mmol} / \mathrm{L}$ sodium fluoride, $1 \mathrm{mmol} / \mathrm{L}$ sodium vanadate, and protease inhibitors]. Extracts treated with calf intestinal alkaline phosphatase (CIP, NEB, Ipswich, MA) were prepared by lysing cells in NP40 lysis buffer lacking sodium vanadate and sodium fluoride. Clarified total cell lysates were quantified using Bio-Rad (Richmond, CA) protein assay kits. Immunoprecipitations were incubated overnight at $4{ }^{\circ} \mathrm{C}$, using 0.5 to $1 \mathrm{mg}$ of total protein lysate, 1 to $2 \mu \mathrm{g}$ affinity-purified antibody, and protein $\mathrm{A}$ or $\mathrm{G}$ agarose beads (Invitrogen). Western blotting was done with 50 to $100 \mu \mathrm{g}$ of protein extract separated by SDS-PAGE and transferred to nitrocellulose membrane (GE Osmonics, Minnetonka, MN). Polyclonal antibody to TSC2 was raised in rabbits (Pocono Rabbit Farms, Canadensis, PA) using a COOH-terminal TSC2 peptide (corresponding to residues 1788-1807) coupled to keyhole limpet hemocyanin as an immunogen, and later affinity-purified (Pierce Biotechnology, Rockford, IL). Rabbit polyclonal antibodies to cyclin D1/2, cyclin D3, CDK6, and p16 were similarly generated by our laboratory. Affinity-purified antibodies to Myc (clone 9E10, Labvision NeoMarkers, Fremont, CA), HA (clone 12CA5, NeoMarkers), Flag (M2, Sigma), cyclins D1, D2, D3 (G124-259, PharMingen, San Diego, CA), tubulin (NeoMarkers), and phosphorylated $\mathrm{Thr}^{389}$ of S6K (Cell Signaling Technologies, Beverly, MA) were purchased commercially.
In vitro kinase assay. For kinase assays, $293 \mathrm{~T}$ cells in a six-well plate were transfected as indicated and serum-starved for 2 to 8 hours. One half of anti-HA-immunoprecipitate was used for Western blot analysis and the other half was washed twice in kinase assay buffer $[20 \mathrm{mmol} / \mathrm{L}$ HEPES (pH 7.5), $10 \mathrm{mmol} / \mathrm{L} \mathrm{MgCl}_{2}, 1 \mathrm{mmol} / \mathrm{L}$ DTT] and incubated for 15 minutes at $30^{\circ} \mathrm{C}$ with $0.5 \mu \mathrm{g}$ glutathione $S$-transferase (GST)-S6 (20), $50 \mu \mathrm{mol} / \mathrm{L}$ of ATP, and $2 \mu \mathrm{Ci}{ }^{32} \mathrm{P}-\gamma$-dATP. Reactions were separated by SDS-PAGE, dried, and visualized by autoradiography.

\section{Results}

Detection of TSC2 as a cyclin D-interacting protein. Studies in Drosophila have shown that cyclin D plays a role in cell growth control $(10,11)$, and that it interacts genetically with TSC2 (21), a negative regulator of cell growth. To test whether TSC2 and cyclin D may physically interact in mammalian cells, 293T cells were transiently transfected with Flag-tagged full-length TSC2 in combination with one of the three D-type cyclins. Anti-Flag immunoprecipitates were resolved by SDS-PAGE and each of the three D-type cyclins were detected in the Flag-TSC2 immunocomplexes (Fig. 1 $A$ ). The cyclin box of cyclin D1 (amino acids 42-153), the region that is sufficient for association with CDK4/6, does not bind to TSC2 (Fig. $1 B$ ). Rather, both the $\mathrm{NH}_{2}$ terminus (amino acids $1-42$, corresponding to the pRb-binding domain) and the $\mathrm{COOH}$ terminus (amino acids 153-295) of cyclin D1 retain the ability to associate with TSC2, consistent with the formation of a ternary complex between TSC2-cyclin D1-CDK4/6. These observations were further supported by results from a yeast three-hybrid screen in which cyclin D1-CDK6 ${ }^{\mathrm{K} 43 \mathrm{M}}$ (catalytically inactive complex) was used as bait to detect interactions with prey from a human HaCaT cell cDNA library. Aside from known cyclin D-CDK4/6 binding partners, such as members of the INK4 and CIP/KIP families, and the pRb family member p130, COOH-terminal truncated forms of TSC2 were isolated 20 times in this screen (M.A. Nichols and Y. Xiong, data not shown). CDK6 was not required for binding of cyclin D1 with TSC2, as cyclin D1 alone positively interacted with TSC2 in a directed yeast-two hybrid assay (data not shown).

TSC2 heterodimerizes with TSC1, and acts to negatively regulate cell growth by inhibiting the protein kinase mTOR (22-24). The TSC1-TSC2 complex is sensitive to growth conditions-in the presence of growth factors and mitogens, TSC2 is multiphosphorylated, rendering TSC1-TSC2 inactive, and in their absence, TSC2 is hypophosphorylated, allowing TSC1-TSC2 to actively repress mTOR (23-30). To determine whether growth conditions might have an effect on the association of cyclin D1 with TSC2, we did coimmunoprecipitation experiments from serum-starved cells with or without subsequent serum stimulation (Fig. $1 C$ ). Consistent with previous observations, we observed more stable detection of TSC2 when its plasmid was coexpressed with one encoding TSC1 (31) Although TSC2 was detectable in the anti-cyclin D1 immunocomplex from serum-starved cells, its association increased as the cells were stimulated with $10 \%$ serum (Fig. 1C, lanes 13-18). TSC1 was also detectable in the cyclin D1 immunocomplex, presumably through its association with TSC2 (Fig. 1C, lanes 14, 16, and 18) Together, these data show an association between cyclin D1 and TSC2 that allows for CDK4/6 binding and is subject to regulation by serum-derived growth signals.

Cyclin D1 overexpression abrogates the growth-inhibitory effects of TSC1-TSC2. In the absence of functional TSC1-TSC2, metazoan cells are quantitatively larger and conversely, excess TSC1-TSC2 causes a measurable decrease in overall cell size $(21,29$, 32,33 ). A physical interaction of cyclin D-CDK with the TSC1-TSC2 


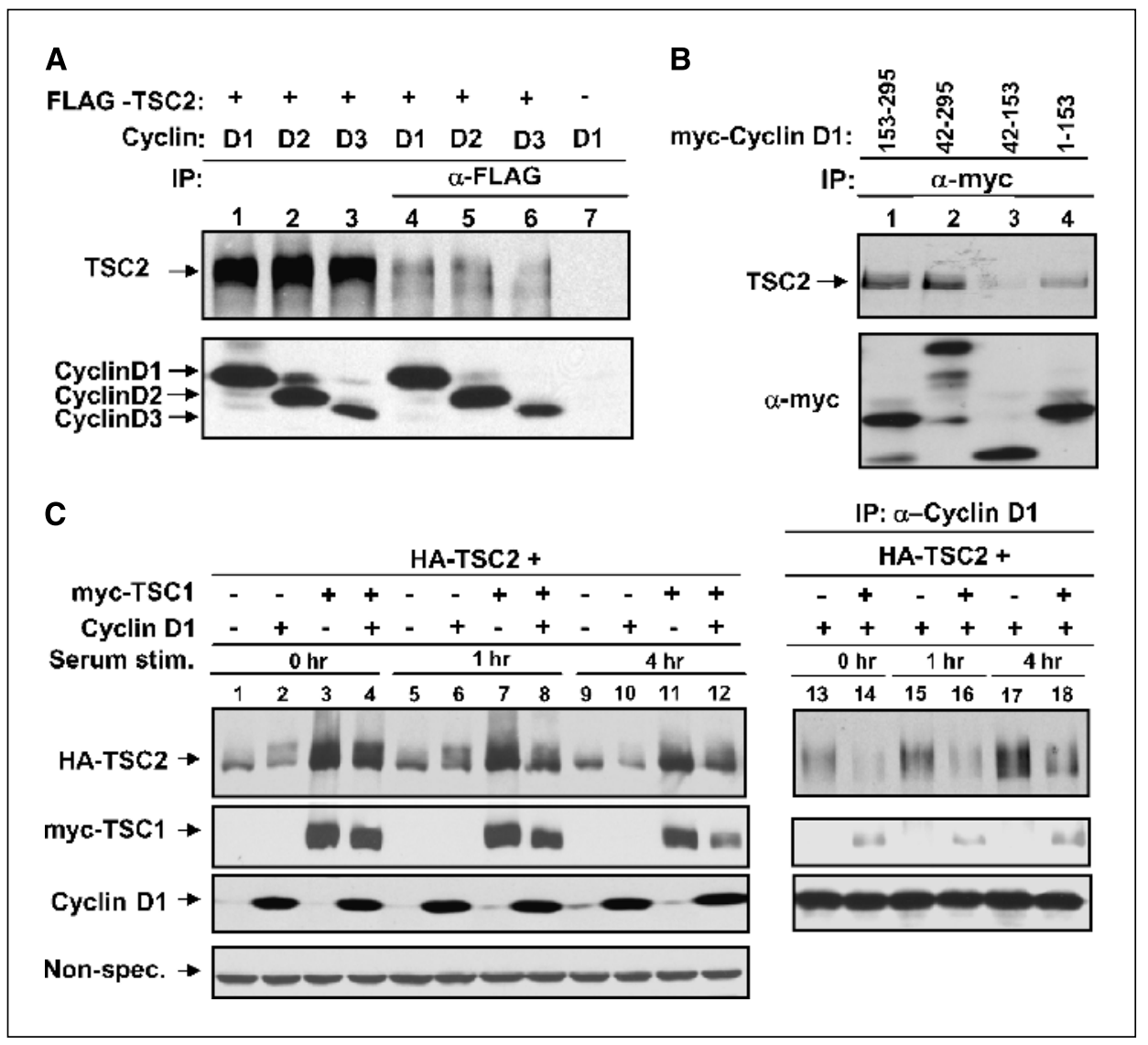

Figure 1. Cyclin $D$ interacts with TSC2. $A$, 293T cells were transfected with plasmids encoding Flag-TSC2 and/or the indicated cyclin D. Whole cell lysate (lanes 1-3) or anti-Flag immunoprecipitates (lanes 4-7) were separated by SDS-PAGE and blotted with antibody against TSC2 (top) or cyclins D1-3 (bottom). $B$, myc-cyclin D1 was divided into three domains (amino acids 1-42, pRb binding; 42-153, cyclin box; $153-295, \mathrm{COOH}$ terminus), expressed in 293T, and immunoprecipitated with anti-myc antibody to test for association with cotransfected HA-TSC2. Equal expression of HA-TSC2 was confirmed in whole cell lysates (data not shown). C, 293T cells were transfected with equal amounts of HA-TSC2 CDNA with or without myc-TSC1 and/or cyclin D1 cDNAs. Following treatment, whole cell lysate (lanes 1-12) or anti-cyclin D1 immunoprecipitates (lanes 13-18) were run on SDS-PAGE and analyzed by Western blot as indicated.

complex implies a role for cyclin D-CDK in cell growth control, consistent with previous conclusions based on Drosophila genetics (21). We therefore evaluated the effect of cyclin D1 expression on TSC1-TSC2 function by using flow cytometry to measure changes in cell size-determined by the forward scatter of light produced by the transfected cells. As expected, when TSC1 and TSC 2 were ectopically expressed in U2OS cells, a decrease in mean forward scatter of $\mathrm{G}_{1}$ phase cells was observed (Fig. $2 A$ ). A similar but less pronounced effect on cell size was evident in $\mathrm{G}_{2}$-M phase cells (data not shown). The addition of cyclin D1 to cells overexpressing TSC1-TSC2 nearly restored the average cell size to control levels (Fig. 2A). TSC1 and TSC2 have also been proposed to affect cell cycle progression $(21,34-36)$. TSC1-TSC2 overexpression caused only subtle alterations in cell cycle distribution, leading to a slight increase in the $G_{1}$ population, which again was abrogated when cyclin D1 was coexpressed (Fig. $2 A$ ).

Mammalian cell growth is regulated by the kinase activity of mTOR directed at either inhibiting or activating, respectively, two primary targets-4E-BP1 and S6K1 (37). The activity of mTOR, in turn, is negatively regulated by the TSC1-TSC2 complex which itself may be controlled in part by cyclin D-CDK4/6 (Fig. $2 A$ ). Therefore, to determine whether the antagonizing effect of cyclin D1 toward the TSC complex is mediated through mTOR activity, we assayed for mTOR-dependent phosphorylation of 4E-BP1 and S6K1 under conditions of cellular proliferation or quiescence. 293T cells were transfected with plasmids encoding either HA-4E-BP1 alone or with cyclin D and CDK4, and were cultured either with $10 \%$ serum or in the complete absence of serum for 1 hour before harvesting. Under normal growth conditions, HA-4E-BP1 can be seen both as a faster migrating nonphosphorylated band and as a more slowly migrating phosphorylated band (Fig. 2B, lane 2). Upon serum withdrawal, HA-4E-BP1 collapses to the nonphosphorylated form (Fig. $2 B$ ). However, under the same conditions, coexpression of cyclin D1-CDK4 substantially prevented the loss of phosphorylated HA-4E-BP1. Serum-induced phosphorylation of 4E-BP1 is dependent on mTOR $(38,39)$, and the repression of mTOR following serum depletion requires the activity of the TSC1-TSC2 complex $(40,41)$. Therefore, these data are consistent with the suggestion that cyclin D1-CDK4 acts to protect the mTOR signaling pathway from TSC1-TSC2 function.

Similar results were obtained when the activity of S6K1 was examined under conditions of serum withdrawal. Plasmid encoding HA-S6K1 was transfected into 293T cells, either independently or in conjunction with cyclin D1-CDK4. Twenty-four hours following transfection, serum was removed and the cells were collected 2,4 , or 8 hours later. HA-S6K was immunoprecipitated from cell lysates and assayed for its ability to phosphorylate GST-S6 (Fig. 2C). Under standard growth conditions, HA-S6K efficiently phosphorylated GST-S6, but as the duration of serum starvation increased, HA-S6K activity diminished (Fig. 2C, lanes 3, 5, and 7). However, when plasmids encoding cyclin D1-CDK4 were coexpressed with HA-S6K, a partial attenuation of the rapid decline in S6K activity was observed at each time point (Fig. $2 C$, lanes 4,6 , and 8 ). Furthermore, whereas overexpression of TSC1 and TSC 2 can reduce the activating phosphorylation of S6K1 at $\mathrm{Thr}^{389}$ by mTOR, the coexpression of cyclin D1, either alone or in combination with CDK6, restores mTOR-dependent phosphorylation of S6K1 (Fig. 2D). Thus, high cyclin D-CDK4/6 levels down-regulate the capacity of the TSC1TSC2 complex, when boosted by either serum depletion or its overexpression, to inhibit mTOR signaling. 
TSC1 and TSC2 are phosphorylated in a CDK-dependent manner. Two lines of evidence suggest that cyclin D-CDK4/6 may promote the phosphorylation of TSC2 and/or TSC1: $(a)$ physical interaction of the TSC1-TSC2 complex with cyclin D-CDK4/6 (Fig. 1) and (b) retarded mobility of both TSC1 and TSC2 on SDSPAGE when cyclin D and CDK4/6 were coexpressed (Fig. 2D). In order to evaluate the potential kinase activity of other CDKs toward TSC2, cDNAs for TSC2 and myc-TSC1 were cotransfected into U2OS cells with different cyclin and CDK combinations, and total cell lysates were resolved by SDS-PAGE (Fig. 3A). As expected, coexpression of cyclin D1 and CDK6 caused a mobility shift of TSC2, whereas the catalytically inactive $\mathrm{CDK} 6^{\mathrm{K} 43 \mathrm{M}}$ mutant failed to alter the mobility of TSC2 (Fig. $3 A$ ). CIP treatment of extracts confirmed that the mobility shift was due to phosphorylation (Fig. 3B). Interestingly, the mobility of TSCl was also retarded by coexpression with cyclin D1-CDK6, but not by cyclin D1-CDK6 ${ }^{\mathrm{K} 43 \mathrm{M}}$. The phosphorylation of TSC1 on at least three sites, T417, S584, and T1047, by cyclin B-CDK1 has been described $(42,43)$. We found that, in fact, several different cyclin-CDK pairs, including cyclin D-CDK4 (data not shown), cyclin E-CDK2, cyclin A-CDK1, and cyclin A-CDK2 promoted the apparent phosphorylation of both TSC1 and TSC2 upon coexpression (Fig. $3 A$ ). These results suggest that both TSC1 and TSC2 can be phosphorylated in a CDK-dependent manner, either directly or indirectly, and offer one potential mechanism for the regulation of the TSC1-TSC2 complex by cyclin-CDK partners.

Down-regulation of TSC1-TSC2 by cyclin $D$. Another notable consequence of the concomitant expression of plasmids encoding cyclin D-CDK6 (wild-type or kinase-dead) and the TSC1-TSC2 complex is a decreased detection of both TSC1 and TSC2 protein levels (Figs. $2 D$ and $3 A$ ). Overexpression of cyclin D1 alone is able to cause the down-regulation of coexpressed myc-TSCl and HA-TSC2, although this effect is dependent on growth conditions (Fig. 1C). HA-TSC2 and myc-TSC1 coexpressed with cyclin D1 in $293 \mathrm{~T}$ cells are most stable under serum-starved conditions, and become increasingly sensitive to cyclin D1-mediated downregulation upon serum restimulation (Fig. 1 , lanes 4, 8, and 12 ). Cyclins D2 and D3 elicit a similar effect on decreasing TSC1-TSC2 under the same assay conditions (data not shown). To determine whether expression of cyclin D1 alone might down-regulate TSC protein levels through the activation of endogenous CDK4/6, we used cyclin $\mathrm{D} 1^{\mathrm{K} 112 \mathrm{E}}$, which is a cyclin box mutant that fails to bind or activate either CDK4 or CDK6 $(44,45)$. As shown in Fig. $4 A$, cyclin $\mathrm{D}^{\mathrm{K} 112 \mathrm{E}}$ is also capable of down-regulating myc-TSC1 and HA-TSC2, demonstrating the kinase-independence of this activity.

For further confirmation of a CDK-independent down-regulation of ectopic TSC1 and TSC2 by coexpressed cyclin D1, we took advantage of the differential inhibitory modes of the two families of CDK inhibitors, the INK4 versus the CIP/KIP family. p16 ${ }^{\mathrm{INK} 4 a}$, which forms a binary complex with $\mathrm{CDK} 4 / 6$, is unable to protect myc-TSC1 or HA-TSC2 from down-regulation by cyclin D1 expression (Fig. $4 B$ ). In contrast, p21 ${ }^{\mathrm{CIP}}$, which forms a ternary complex with cyclin D-CDK4/6, is able to attenuate the down-regulation of myc-TSC1 and HA-TSC2 caused by either cyclin D1 alone or cyclin D1-CDK6 (Fig. 4B). A 3-fold increase in transfected cyclin D1
Figure 2. Cyclin D-CDK4/6 abrogates the growth-inhibitory effects of TSC1-TSC2. $A$, cell size distribution of $\mathrm{G}_{1}$ phase cells. U2OS were transfected with GFP-spectrin alone (control), or with GFP-spectrin, TSC1, and TSC2, with or without cyclin D1, and analyzed 48 hours later by flow cytometry to assess cell cycle and cell size (FSC) profiles. Results are representative of three independent experiments.

$B, \mathrm{HA}-4 \mathrm{E}-\mathrm{BP} 1$ was transiently expressed in 293T alone, or with cyclin D1 and CDK4. After 24 hours, cells were either left untreated, or were starved of serum for 1 hour prior to harvest. Whole cell lysates were run on SDS-PAGE and blotted with anti-HA antibody. $C, 293$ T cells were transfected with empty vector or HA-S6K, with or without cyclin D1 and CDK4, and cultured for 24 hours under normal growth conditions. Before harvest, cells were either left untreated ( 0 hours), or were serum-starved for 2 , 4 , or 8 hours. HA-S6K was immunoprecipitated from each total cell lysate; one quarter was separated by SDS-PAGE and probed with anti-HA antibody (bottom), and the remainder of each immunoprecipitation was used in a kinase assay with purified GST-S6 and ${ }^{32}$ P-ATP, separated by SDS-PAGE, and analyzed by autoradiography (top). The band intensities of ${ }^{32} \mathrm{P}$-GST-S6 were quantified by densitometric analysis, and represented as relative units (bottom). $D$, U20S were transfected with the indicated plasmids, harvested 48 hours later, and analyzed by SDS-PAGE and Western blotting with anti-HA, anti-myc, and anti-phosph-T389-S6K antibodies.

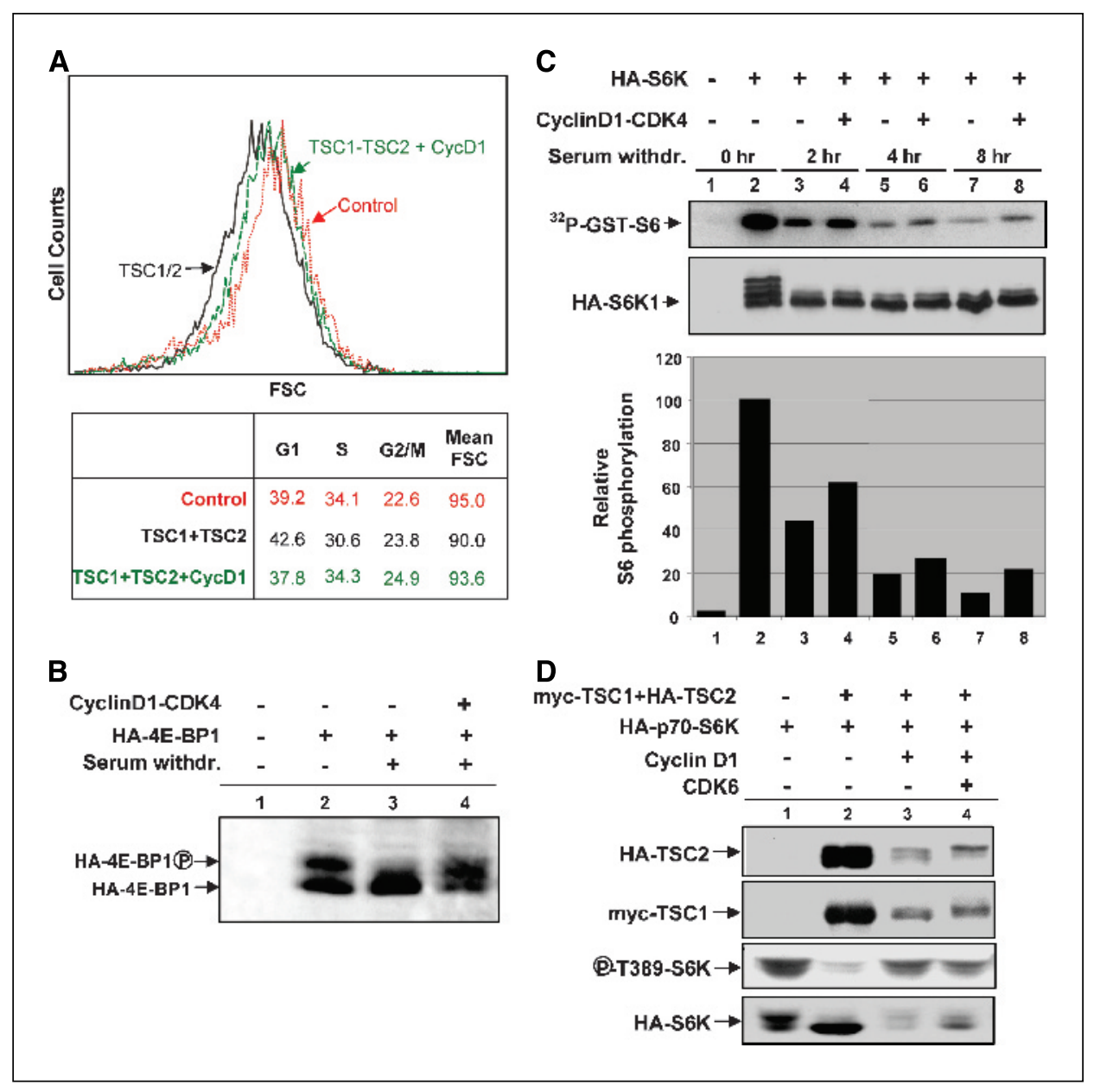




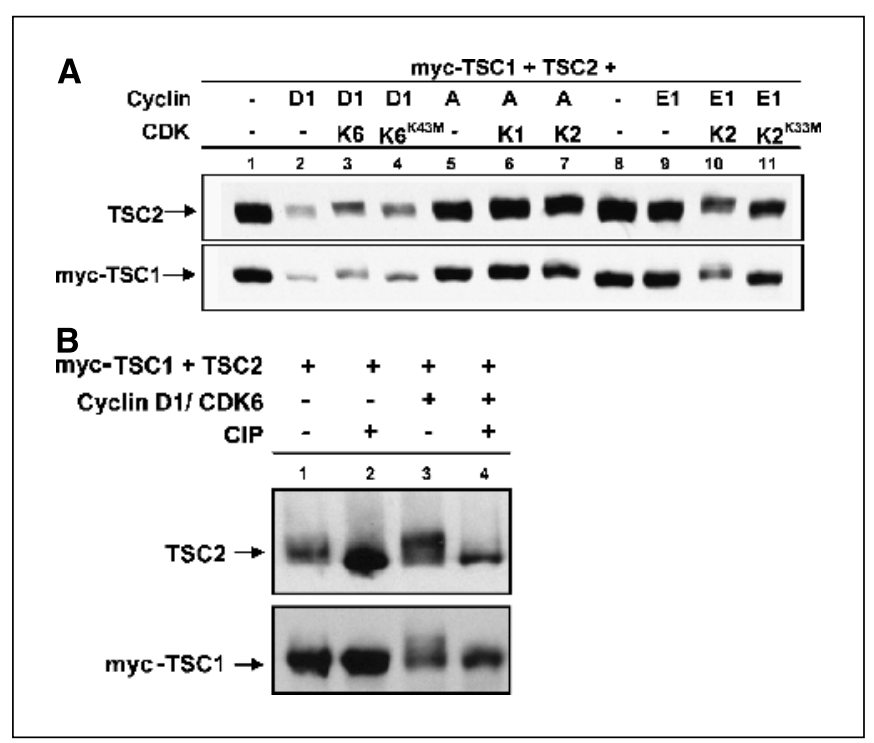

Figure 3. CDKs promote the phosphorylation of TSC1 and TSC2. A, U2OS were transfected with plasmids encoding myc-TSC1 and TSC2, alone or in conjunction with various cyclin-CDK combinations, and whole cell lysates were separated by SDS-PAGE and analyzed by Western blot. $B$, U2OS were transfected with the indicated plasmids and analyzed as in $(A)$, except that each whole cell lysate was either treated with 25 units of CIP, or left untreated.

expression plasmid causes a correspondingly larger decrease in both myc-TSC1 and TSC2 levels (Fig. 4C), indicating that the effect of cyclin D1 on TSC1 and TSC2 protein levels is equivalent. We failed to inhibit cyclin D1-mediated TSC1-TSC2 down-regulation with MG132, chloroquine, and leupeptin (data not shown), ruling out the potential involvement of the proteasome, lysosome, or calpain. Detection of either TSC1 or TSC2 is increased when coexpressed with the second TSC complex member (Fig. $1 C$ ), suggesting that proper folding or protein stability requires TSC1-TSC2 complex formation. TSC1 and, to a lesser extent, TSC2, fractionate in an NP40-insoluble fraction when expressed alone, and are found predominantly in an NP40-soluble fraction when coexpressed (Fig. $4 C$; ref. 46). Cyclin D1 expression causes a dose-dependent shift of both TSC1 and TSC2 from the NP40-soluble fraction to the insoluble fraction (Fig. $4 C$ ). In addition, binding of ectopically expressed TSC2 and cyclin $\mathrm{D} 1$ is diminished by coexpression of TSC1 (Fig. 1C, lanes 14, 16, and 18), indicating that stable TSC1-TSC2 complex formation is incompatible with cyclin D1 association. Together, these data suggest that cyclin D1 overexpression is able to negatively regulate coexpressed TSC1 and TSC2 via a CDK-independent mechanism, possibly through complex disruption.

\section{Discussion}

The "pRb pathway" controls the transition from the $G_{1}$ to the $S$ phase of the cell cycle and is often denoted as a linear sequence of interactions: INK4 proteins-I cyclin D-dependent kinases $\rightarrow p R b$ family proteins (47). The importance of this pathway is underscored by the likelihood that it is deregulated in most, if not all, human tumors. Interestingly, the aberrant activation of CDK4/6, either by loss of INK4 inhibitor genes or by amplification and overexpression of cyclin D, CDK4, or CDK6 genes, is observed in diverse cancers disproportionately more often than is inactivation of $\mathrm{pRb}$ proteins. This observation suggests that inappropriate cyclin D-CDK4/6 activity confers greater growth advantages to cells than mere loss of $\mathrm{pRb}$ function, and implies that inactivation of cyclin D-CDK4/6 targets in addition to $\mathrm{pRb}$ may contribute to its oncogenic potency (4). We have shown that cyclin D, either alone or in conjunction with CDK4/6 can physically interact with the tumor suppressor protein, TSC2, and down-regulate the growthsuppressive function of the TSC1-TSC2 heterodimer.

TSC1 and TSC2 were originally identified as two separate loci linked to the congenital disorder, tuberous sclerosis complex (TSC, refs. 48, 49). The in vivo function of TSC1-TSC2 had been difficult to pinpoint until studies in Drosophila melanogaster identified the

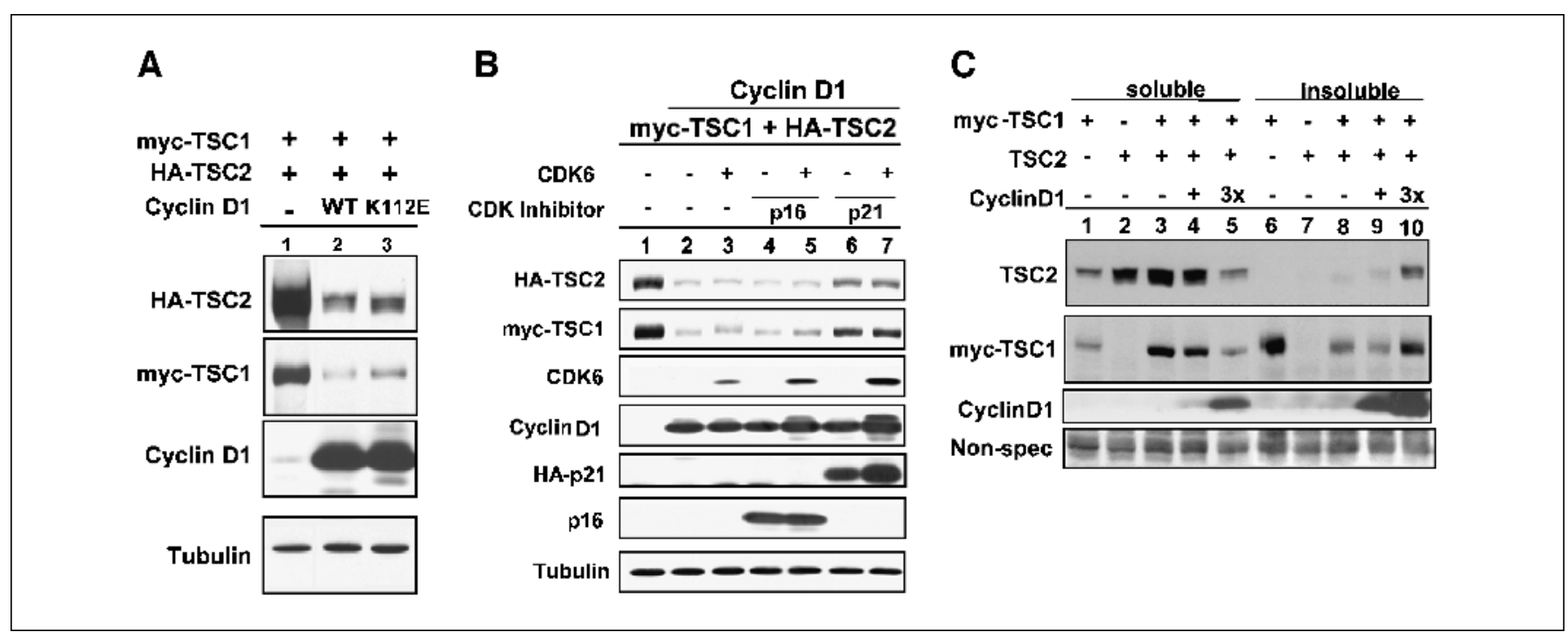

Figure 4. Down-regulation of TSC1-TSC2 by cyclin D. A, myc-TSC1 and HA-TSC2 were ectopically expressed in U2OS alone, or in conjunction with wild-type or mutant (K112E) cyclin D1. Total cell lysates were analyzed by SDS-PAGE and Western blot, as indicated. $B$, U2OS were transiently transfected with plasmids encoding HA-TSC2 and myc-TSC1, along with cyclin D1 and/or CDK6, and p16 or HA-p21, as indicated, and analyzed by SDS-PAGE and Western blot, probing with anti-HA, -myc, -CDK6, -cyclin D1, -p16, and -tubulin antibodies. C, U2OS were transiently transfected with $100 \mathrm{ng}$ each of pcDNA3-myc-TSC1 and pcDNA3-HA-TSC2 alone, or with 100 or $300 \mathrm{ng}(3 \times)$ pcDNA3-cyclin D1. Transfected cells were collected and lysed in NP40 lysis buffer (soluble fraction), and the remaining pellet (insoluble fraction) was solubilized in a SDS lysis buffer. The resulting samples were separated by SDS-PAGE and analyzed by Western blot. 
TSC genes (dTSC1 and dTSC2) as important regulators of cell growth $(21,32,35,50)$. Numerous studies, both in Drosophila and mammalian systems, have linked TSC1-TSC2 with several other gene products involved in the insulin $\rightarrow$ phosphoinositide-3-kinase$\rightarrow$ AKT $\rightarrow$ TOR cell growth signaling network (51). The TSC1-TSC2 complex is a key negative regulator of TOR kinase activity, integrating diverse inputs from growth factors, oxygen and nutrient availability, and energy status. The TOR inhibitory activity of TSC1-TSC2 stems from the GTPase-activating protein domain of TSC2, which inactivates the GTPase Rheb and prevents it from stimulating the kinase activity of mTOR (52-58).

The coupling of cell division with cell growth in multicellular organisms intuitively requires more complex regulatory mechanisms than those used by unicellular organisms (59). Whereas unicellular organisms must continually adapt to changing nutrient and energy availability, cells of a muticellular organism are maintained within an environment of relatively constant and abundant nutrient and energy supply, and rely on extracellular cues, in the form of mitogenic or growth factor signaling, to drive cell division or cell growth, respectively. Cyclins are regarded as potential "cell growth sensors" or "translational sizers" that may transmit growth stimuli to proliferative pathways because they are rate-limiting for cell cycle progression and their expression, or accumulation, is sensitive to the rate of protein synthesis $(3,60)$. Cyclin $\mathrm{D}$ might be considered a bilateral relay for proliferation signals because its activation of CDK is derived from mitogens and/or growth factors and because it can simultaneously promote cell cycle progression as well as cell growth (Fig. 5).

We have shown that the TSC1-TSC2 heterodimer may be a molecular target of cyclin D-CDK's growth control activity. An analogous association has also been observed in Drosophila in which $d T S C 1$ and $d T S C 2$ have been found to interact genetically with $c y c D$ and $C D K 4$ (21). Although dTSC1-dTSC2 overexpressed in the eye resulted in a pronounced reduction in overall eye size, co-overexpression of cyclin D-CDK4 rescued this defect. Interestingly, cyclin $\mathrm{E}$ co-overexpression was also capable of rescuing the small eye phenotype, and reduction in cyclin E or cyclin A levels resulted in even smaller eyes. These studies provide a premise to

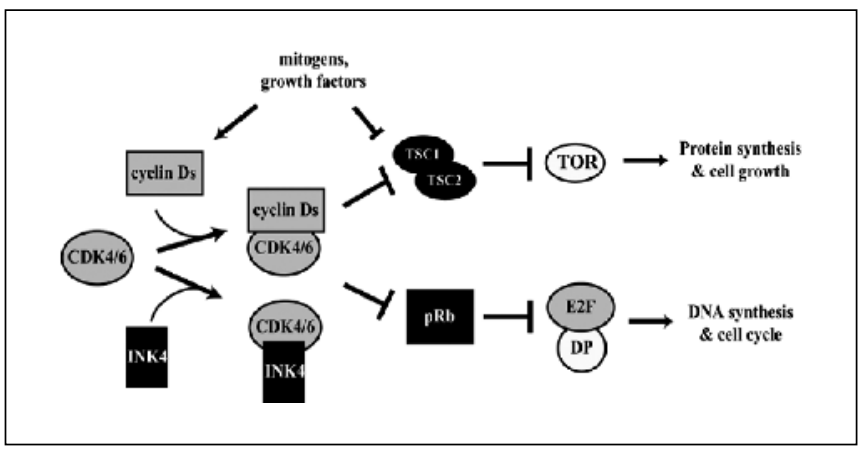

Figure 5. Model of cyclin D-CDK4/6 activity in cell division and growth. Early in $\mathrm{G}_{1}$, mitogenic and growth factor signaling induce the synthesis of $D$-type cyclins, leading to activation of cyclin D-CDK4/6. The INK4 family of CDK inhibitor proteins specifically inhibits CDK4/6. When activated, cyclin Ds-CDK4/6 and cyclin Es-CDK2 (not shown here) cooperatively phosphorylate $\mathrm{pRb}$ family proteins, derepressing E2F to allow transcription of E2F target genes, thereby permitting $\mathrm{G}_{1}$ to $S$ transition. In concert, cyclin $\mathrm{D}-\mathrm{CDK} 4 / 6$ may also inactivate the TSC1-TSC2 heterodimer, thereby activating the TOR growth-signaling pathway. Thus, cyclin D-CDK4/6 may be seen as a bilateral relay for proliferation signals, integrating mitogenic and growth factor signaling to stimulate DNA synthesis as well as protein synthesis, thereby driving both the cell cycle and cell growth. Gray, proto-oncogenic proteins; black, tumor suppressor proteins. our findings that in mammalian cells, cyclin D-CDK could act upstream of, and inhibitory to, TSC1-TSC2. Additionally, the studies in Drosophila also suggest the potential involvement of other cyclins in negatively regulating TSC1-TSC2. Consistent with this, we have detected interactions between TSC2 and cyclins A, B, and E (data not shown), and interactions between TSC2 and cyclin A, cyclin B, and CDK1 have been reported previously $(42,61)$.

The coexpression of TSC1-TSC2 with multiple cyclin-CDK complexes, but not with kinase-dead CDK complexes, results in the phosphorylation of both TSC1 and TSC2 (Fig. 3). Although in additional experiments, we have failed to unambiguously show direct phosphorylation of TSC1 or TSC2 by cyclin D1-CDK4/6 (data not shown), we cannot exclude this possibility. Astrinidis et al. described the direct phosphorylation of TSC1 by cyclin B-CDK1 in nocodazole-treated cells (42). The shared ability of multiple cyclinCDK pairs to bind to, and possibly phosphorylate, TSC1-TSC2 suggests that throughout all stages of the cell cycle, the TSC complex is under the negative regulation of cyclin-CDK complexes, ensuring continuous protein synthesis and growth to meet the needs of a dividing cell. In fact, early studies have shown that the growth of metazoan cells is not confined to a single phase of the cell cycle but rather is continuous throughout (62).

The down-regulation, or decrease in detection, of TSC1 and TSC2 does not require their phosphorylation promoted by the coexpression of cyclin Ds-CDK4/6, nor does it require binding of CDK4/6 by cyclin D1 (Figs. 3 and 4). Cyclin D, therefore, seems to affect TSC1 and TSC2 through both kinase-dependent and kinase-independent mechanisms. The cyclin D-dependent downregulation of TSC1-TSC2 coincides with their shift from a soluble to an insoluble fraction of cell lysate, consistent with a reduced ability of the TSC1 and TSC2 monomers to form a dimeric complex (46). Because the effect of cyclin D overexpression on TSC1 and TSC2 steady state levels was most pronounced on ectopically expressed proteins (data not shown), these data suggest that cyclin D might interfere with the folding of nascent TSC1 or TSC2, thereby preventing stable complex formation. The effect, if any, of cyclin D-CDK-driven phosphorylation of TSC1 and TSC2 might be masked by the CDK-independent ability of cyclin D to down-regulate the TSC1-TSC2 complex.

In summary, we have described the inhibition of the tumor suppressor complex TSC1-TSC2, a key negative regulator of mTOR activity, by cyclin D1, itself a well-known proto-oncogene frequently amplified in various types of human cancers. mTOR stimulates cell growth through ribosome biogenesis and activity, and the importance of its regulation in suppressing unrestrained cell growth and proliferation is manifest in many cancers (63). Further study of the cellular setting(s) and mechanism(s) whereby cyclin D1, with or without its CDK partners, is able to antagonize TSC1TSC2 activity will be crucial to our understanding of the interplay between cell cycle and cell growth regulation, and may validate the targeting of mTOR in tumors in which cyclin D1 is overexpressed.

\section{Acknowledgments}

Received 6/24/2005; revised 9/26/2005; accepted 10/12/2005.

Grant support: NIH grant CA68377 (Y. Xiong). S.D. Shumway is supported by a postdoctoral fellowship from the American Cancer Society.

The costs of publication of this article were defrayed in part by the payment of page charges. This article must therefore be hereby marked advertisement in accordance with 18 U.S.C. Section 1734 solely to indicate this fact.

We thank Michael A. Nichols for initial detection of the cyclin D-TSC2 interaction in a yeast three-hybrid screen, Paula Miliani de Marval for critical reading of the manuscript, Yizhou He for technical assistance, and other members of the Xiong laboratory for helpful discussions. 


\section{References}

1. Nurse P. Genetic control of cell size at cell division in yeast. Nature 1975;256:547-51.

2. Hartwell LH, Unger MW. Unequal division in Saccharomyces cerevisiae and its implications for the control of cell division. J Cell Biol 1977;75:422-35.

3. Jorgensen P, Tyers $M$. How cells coordinate growth and division. Curr Biol 2004;14:R1014-27.

4. Deshpande A, Sicinski P, Hinds PW. Cyclins and cdks in development and cancer: a perspective. Oncogene 2005;24:2909-15.

5. Shamji AF, Nghiem P, Schreiber SL. Integration of growth factor and nutrient signaling: implications for cancer biology. Mol Cell 2003;12:271-80.

6. Pei XH, Xiong Y. Biochemical and cellular mechanisms of mammalian CDK inhibitors: a few unresolved issues. Oncogene 2005;24:2787-95.

7. Franklin DS, Xiong Y. Induction of pl8INK4c and its predominant association with CDK4 and CDK6 during myogenic differentiation. Mol Biol Cell 1996;7:1587-99.

8. Zwicker J, Brusselbach S, Jooss KU, et al. Functiona domains in cyclin D1: pRb-kinase activity is no essential for transformation. Oncogene 1999;18:19-25.

9. Yu BD, Becker-Hapak M, Snyder EL, et al. Distinct and nonoverlapping roles for $\mathrm{pRB}$ and cyclin $\mathrm{D}$ : cyclindependent kinases 4/6 activity in melanocyte survival. Proc Natl Acad Sci U S A 2003;100:14881-6.

10. Meyer CA, Jacobs HW, Datar SA, et al. Drosophila Cdk4 is required for normal growth and is dispensable for cell cycle progression. EMBO J 2000;19:4533-42.

11. Datar SA, Jacobs HW, de la Cruz AF, Lehner CF, Edgar BA. The Drosophila cyclin D-Cdk4 complex promotes cellular growth. EMBO J 2000;19:4543-54.

12. Kozar K, Ciemerych MA, Rebel VI, et al. Mouse development and cell proliferation in the absence of D-cyclins. Cell 2004;118:477-91.

13. Malumbres M, Sotillo $R$, Santamaria $D$, et al. Mammalian cells cycle without the D-type cyclin dependent kinases Cdk4 and Cdk6. Cell 2004;118 493-504.

14. Martin J, Hunt SL, Dubus P, et al. Genetic rescue of Cdk4 null mice restores pancreatic $\beta$-cell proliferation but not homeostatic cell number. Oncogene 2003;22: 5261-9.

15. Xin S, Weng L, Xu J, Du W. The role of RBF in developmentally regulated cell proliferation in the eye disc and in cyclin $\mathrm{D} / \mathrm{Cdk} 4$ induced cellular growth Development 2002;129:1345-56.

16. Frei C, Galloni M, Hafen E, Edgar BA. The Drosophila mitochondrial ribosomal protein $\mathrm{mRpL} 12$ is required fo cyclin D/Cdk4-driven growth. EMBO J 2005;24:623-34.

17. Frei C, Edgar BA. Drosophila cyclin D/Cdk4 requires Hif-1 prolyl hydroxylase to drive cell growth. Dev Cell 2004;6:241-51.

18. Matsuura I, Denissova NG, Wang G, et al. Cyclin dependent kinases regulate the antiproliferative function of Smads. Nature 2004;430:226-31.

19. Fu M, Wang C, Li Z, Sakamaki T, Pestell RG. Minireview: Cyclin D1: normal and abnormal functions. Endocrinology 2004;145:5439-47.

20. Pearson RB, Dennis PB, Han JW, et al. The principal target of rapamycin-induced p70s6k inactivation is a novel phosphorylation site within a conserved hydrophobic domain. EMBO J 1995;14:5279-87.

21. Tapon N, Ito N, Dickson BJ, Treisman JE, Hariharan IK. The Drosophila tuberous sclerosis complex gene homologs restrict cell growth and cell proliferation. Cell 2001;105:345-55.

22. Gao X, Zhang Y, Arrazola P, et al. Tsc tumour suppressor proteins antagonize amino acid-TOR signalling. Nat Cell Biol 2002;4:699-704.

23. Inoki $\mathrm{K}, \mathrm{Li} \mathrm{Y}$, Zhu $\mathrm{T}$, Wu J, Guan KL. TSC2 is phosphorylated and inhibited by Akt and suppresses mTOR signalling. Nat Cell Biol 2002;4:648-57.
24. Tee AR, Fingar DC, Manning BD, et al. Tuberous sclerosis complex-1 and -2 gene products function together to inhibit mammalian target of rapamycin (mTOR)-mediated downstream signaling. Proc Natl Acad Sci U S A 2002;99:13571-6.

25. Han S, Santos TM, Puga A, et al. Phosphorylation of tuberin as a novel mechanism for somatic inactivation of the tuberous sclerosis complex proteins in brain lesions. Cancer Res 2004;64:812-6.

26. Li Y, Inoki K, Vacratsis P, Guan KL. The p38 and MK2 kinase cascade phosphorylates tuberin the tuberous sclerosis 2 gene product, and enhances its interaction with 14-3-3. J Biol Chem 2003;278:13663-71.

27. Ma L, Chen Z, Erdjument-Bromage H, Tempst P, Pandolfi PP. Phosphorylation and functional inactivation of TSC2 by Erk implications for tuberous sclerosis and cancer pathogenesis. Cell 2005;121:179-93.

28. Manning BD, Tee AR, Logsdon MN, Blenis J, Cantley LC. Identification of the tuberous sclerosis complex-2 tumor suppressor gene product tuberin as a target of the phosphoinositide 3-kinase/akt pathway. Mol Cell 2002;10:151-62.

29. Potter CJ, Pedraza LG, Xu T. Akt regulates growth by directly phosphorylating Tsc2. Nat Cell Biol 2002;4: 658-65.

30. Roux PP, Ballif BA, Anjum R, Gygi SP, Blenis J. Tumorpromoting phorbol esters and activated Ras inactivate the tuberous sclerosis tumor suppressor complex via p90 ribosomal S6 kinase. Proc Natl Acad Sci U S A 2004; 101:13489-94.

31. Benvenuto G, Li S, Brown SJ, et al. The tuberous sclerosis-1 (TSC1) gene product hamartin suppresses cell growth and augments the expression of the TSC2 product tuberin by inhibiting its ubiquitination. Oncogene 2000;19:6306-16.

32. Gao X, Pan D. TSC1 and TSC2 tumor suppressors antagonize insulin signaling in cell growth. Genes Dev 2001;15:1383-92.

33. Rosner M, Hofer K, Kubista M, Hengstschlager M. Cell size regulation by the human TSC tumor suppressor proteins depends on PI3K and FKBP38. Oncogene 2003;22:4786-98.

34. Miloloza A, Rosner M, Nellist M, et al. The TSC1 gene product hamartin, negatively regulates cell proliferation. Hum Mol Genet 2000;9:1721-7.

35. Potter CJ, Huang H, Xu T. Drosophila Tscl functions with Tsc2 to antagonize insulin signaling in regulating cell growth cell proliferation, and organ size. Cell 2001; 105:357-68.

36. Soucek T, Pusch O, Wienecke R, DeClue JE, Hengstschlager M. Role of the tuberous sclerosis gene2 product in cell cycle control. Loss of the tuberou sclerosis gene- 2 induces quiescent cells to enter $\mathrm{S}$ phase. J Biol Chem 1997;272:29301-8.

77. Fingar DC, Salama S, Tsou C, Harlow E, Blenis J. Mammalian cell size is controlled by mTOR and it downstream targets S6K1 and 4EBP1/eIF4E. Genes Dev 2002;16:1472-87.

38. Burnett PE, Barrow RK, Cohen NA, Snyder SH, Sabatini DM. RAFT1 phosphorylation of the translational regulators p70 S6 kinase and 4E-BP1. Proc Natl Acad Sci U S A 1998;95:1432-7.

39. Gingras AC, Kennedy SG, O'Leary MA, Sonenberg N, Hay N. 4E-BP1 a repressor of mRNA translation, is phosphorylated and inactivated by the Akt(PKB) signaling pathway. Genes Dev 1998;12:502-13.

40. Kwiatkowski DJ, Zhang H, Bandura JL, et al. A mouse model of TSC1 reveals sex-dependent lethality from liver hemangiomas and up-regulation of p70S6 kinase activity in Tscl null cells. Hum Mol Genet 2002;11: 525-34.

41. Jaeschke A, Hartkamp J, Saitoh M, et al. Tuberou sclerosis complex tumor suppressor-mediated S6 kinase inhibition by phosphatidylinositide-3-OH kinase is mTOR independent. J Cell Biol 2002;159:217-24.
42. Astrinidis A, Senapedis W, Coleman TR, Henske EP. Cell cycle-regulated phosphorylation of hamartin the product of the tuberous sclerosis complex 1 gene, by cyclin-dependent kinase 1/cyclin B. J Biol Chem 2003; 278:51372-9.

43. Ballif BA, Roux PP, Gerber SA, et al. Quantitative phosphorylation profiling of the ERK/p90 ribosomal S6 kinase-signaling cassette and its targets the tuberous sclerosis tumor suppressors. Proc Natl Acad Sci U S A 2005;102:667-72.

44. Zwijsen RM, Wientjens E, Klompmaker R, et al. CDKindependent activation of estrogen receptor by cyclin D1. Cell 1997;88:405-15.

45. Inoue K, Sherr CJ. Gene expression and cell cycle arrest mediated by transcription factor DMP1 is antagonized by D-type cyclins through a cyclin-dependent-kinase-independent mechanism. Mol Cell Biol 1998;18:1590-600.

46. Nellist $M$, Verhaaf $B$, Goedbloed $\mathrm{MA}$, et al. TSC2 missense mutations inhibit tuberin phosphorylation and prevent formation of the tuberin-hamartin complex. Hum Mol Genet 2001;10:2889-98.

47. Sherr CJ, McCormick F. The RB and p53 pathways in cancer. Cancer Cell 2002;2:103-12.

48. Consortium TECTS. Identification and characterization of the tuberous sclerosis gene on chromosome 16 The European Chromosome 16 Tuberous Sclerosis Consortium. Cell 1993;75:1305-15.

49. van Slegtenhorst $M$, de Hoogt R, Hermans $C$, et al. Identification of the tuberous sclerosis gene TSC1 on chromosome 9q34. Science 1997;277:805-8.

50. Ito N, Rubin GM. gigas, a Drosophila homolog of tuberous sclerosis gene product-2, regulates the cell cycle. Cell 1999;96:529-39.

51. Inoki K, Ouyang H, Li Y, Guan KL. Signaling by target of rapamycin proteins in cell growth control. Microbiol Mol Biol Rev 2005;69:79-100.

52. Zhang Y, Gao X, Saucedo LJ, et al. Rheb is a direct target of the tuberous sclerosis tumour suppressor proteins. Nat Cell Biol 2003;5:578-81.

53. Stocker $H$, Radimerski $T$, Schindelholz B, et al. Rheb is an essential regulator of $\mathrm{S} 6 \mathrm{~K}$ in controlling cell growth in Drosophila. Nat Cell Biol 2003;5:559-65.

54. Tee AR, Manning BD, Roux PP, Cantley LC, Blenis J. Tuberous sclerosis complex gene products Tuberin and Hamartin, control mTOR signaling by acting as a GTPase-activating protein complex toward Rheb. Curr Biol 2003;13:1259-68.

55. Saucedo LJ, Gao X, Chiarelli DA, et al. Rheb promotes cell growth as a component of the insulin/TOR signalling network. Nat Cell Biol 2003;5:566-71.

56. Li Y, Inoki K, Guan KL. Biochemical and functional characterizations of small GTPase Rheb and TSC2 GAP activity. Mol Cell Biol 2004;24:7965-75.

57. Inoki K, Li Y, Xu T, Guan KL. Rheb GTPase is a direct target of TSC2 GAP activity and regulates mTOR signaling. Genes Dev 2003;17:1829-34.

58. Garami A, Zwartkruis FJ, Nobukuni T, et al Insulin activation of Rheb a mediator of mTOR/S6K/4E-BP signaling, is inhibited by TSC1 and 2. Mol Cell 2003;11: 1457-66.

59. Conlon I, Raff M. Size control in animal development. Cell 1999;96:235-44.

60. Fingar DC, Blenis J. Target of rapamycin (TOR): an integrator of nutrient and growth factor signals and coordinator of cell growth and cell cycle progression. Oncogene 2004;23:3151-71.

61. Catania MG, Mischel PS, Vinters HV. Hamartin and tuberin interaction with the $\mathrm{G} 2 / \mathrm{M}$ cyclin-dependent kinase CDKl and its regulatory cyclins $\mathrm{A}$ and $\mathrm{B}$. J Neuropathol Exp Neurol 2001;60:711-23.

62. Mitchison JM. Growth during the cell cycle. Int Rev Cytol 2003;226:165-258.

63. Ruggero D, Pandolfi PP. Does the ribosome translate cancer? Nat Rev Cancer 2003;3:179-92. 